\title{
Heartbeat: Patient-prosthesis mismatch in adults with congenital heart disease
}

Catherine M Otto

Patient-prosthetic mismatch (PPM) occurs when the effective flow area for a normally functioning prosthetic valve is smaller than needed to provide an adequate cardiac output at rest and with exercise in that specific patient. Current recommendations for avoiding PPM focus on implantation of a valve adequate to the patient's body size. There has been little attention to avoiding PPM by ensuring the patient's body size is appropriate, an approach that may become relevant with the increasing prevalence of obesity in many countries. PPM is a particular problem in patients with congenital heart disease because the patient's body size often continues to increase, due to normal growth, after the prosthetic valve is implanted. Thus, an adequate size prosthetic valve at the time of implantation may be too small once the patient reaches adult stature (figure 1). PPM has deleterious physiologic consequences and may be associated with adverse clinical outcomes. In adults with acquired valve disease, aortic valve PPM is associated with persistent left ventricular hypertrophy, reduced exercise capacity and higher mortality rates in some patient subsets. However, the impact of PPM in adults with congenital heart disease (ACHD) has not previously been studied.

In a Dutch registry of ACHD patients with prosthetic heart valves, Pieper and colleagues (see page 107) found that PPM was present in $42 \%$ of 207 ACHD patients with a prosthetic aortic valve. Severe PPM, defined as an indexed effective orifice area $\leq 0.65 \mathrm{~cm}^{2} / \mathrm{m}^{2}$, was present in 23\% and moderate PPM, defined as an indexed orifice area 0.66 to $0.85 \mathrm{~cm}^{2} / \mathrm{m}^{2}$, was present in $19 \%$ of this cross-sectional cohort. Patients with PPM had a lower exercise capacity compared to those without PPM. On multivariable analysis, PPM remained a significant predictor of exercise capacity along with chronoscopic incompetence, even when LV ejection fraction, haemoglobin level and body surface area were considered. However, despite the association of PPM with diminished

Correspondence to Professor Catherine M Otto, Division of Cardiology, University of Washington, Seattle, WA 98195, USA; cmotto@u.washington.edu exercise capacity, there was no significant difference in death or hospitalization over a mean follow-up of 2.6 \pm 1.1 years between those with and without PPM. It is possible that an effect on outcomes was missed due to the small sample size and short time course of this study; thus we look forward to larger studies with longterm follow-up to further address the impact of PPM in ACHD patients.

The challenges of PPM in ACHD patients are discussed in an editorial by Dr. Hernandez-Vaquero. (see page 89) As he summarizes: "The choice of the appropriate prosthesis for a young patient is likely the easiest way to avoid mismatch. Even when it is not expected to have PPM in that moment, many of them can develop it in the future. Procedures to enlarge the aortic annulus, the implantation of an autologous graft, which has the potential to grow with the patient or, when possible, repair the aortic valve, are other ways to avoid PPM, but decision-making for these techniques is more difficult since they may involve an increased surgical risk" (figure 1). We agree with his suggestion that "Future research should work to establish the mortality pattern, the risk of sudden death and lifethreatening situations during the follow-up of ACHD with severe PPM and its impact on their quality of life."

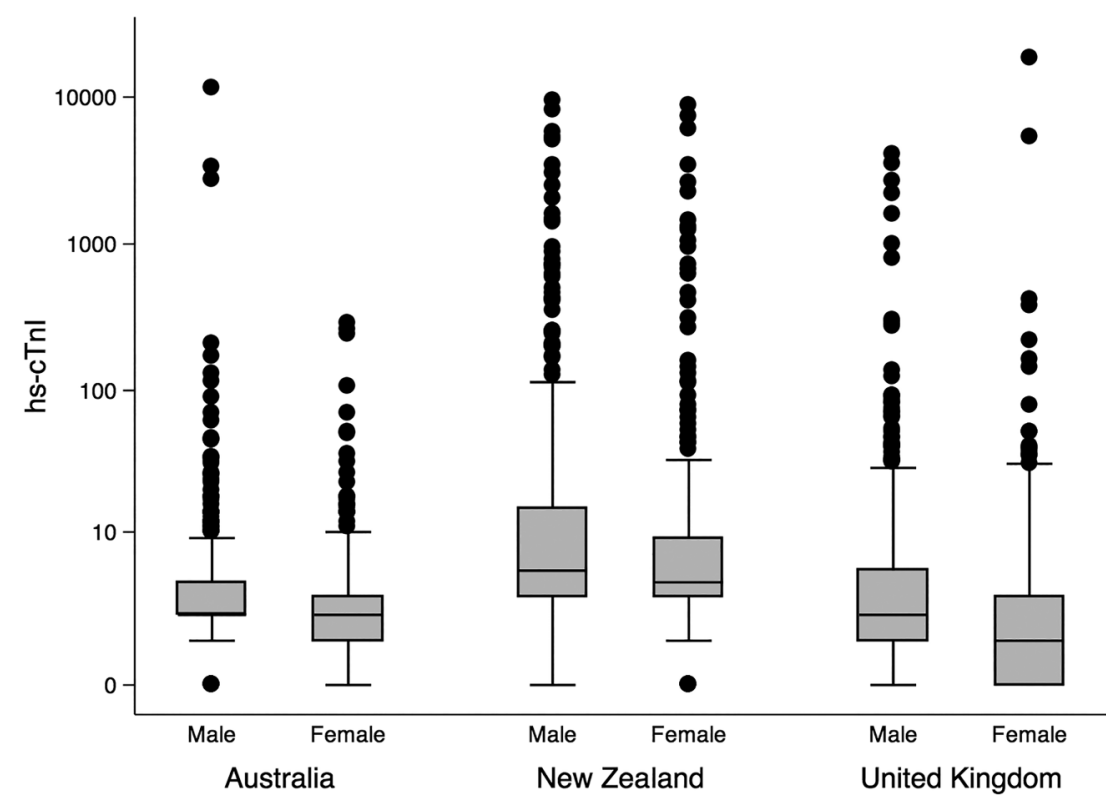

Figure 2 hs-cTnl for males and females by hospital. Data are presented on a logarithmic scale.

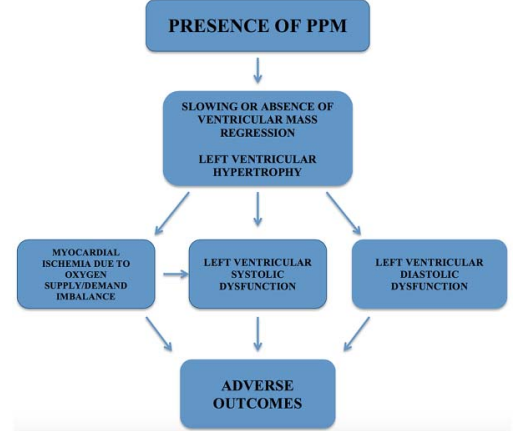

Figure 1 Pathophysiological mechanisms of patient prosthesis mismatch (PPM).

Currently, a single set of cut points for serum levels of high sensitivity troponin (hs-cTnI) are used in men and women presenting with symptoms suggestive of an acute coronary syndrome (ACS). In a multicenter observational study of 2841 patients from Australia, New Zealand and the United Kingdom, adverse cardiac events (MACE) at one-year follow-up occurred in $103(8.7 \%)$ of 1180 women and 234 (14\%) of 1661 men. (see page 120) Compared to a sex-neutral cut point of $26 \mathrm{ng} / \mathrm{L}$, use of lower 99 th percentile cut point of $16 \mathrm{ng} / \mathrm{L}$ in women, reclassified $25(2 \%)$ from normal to an elevated troponin with $7(28 \%)$ of these women 


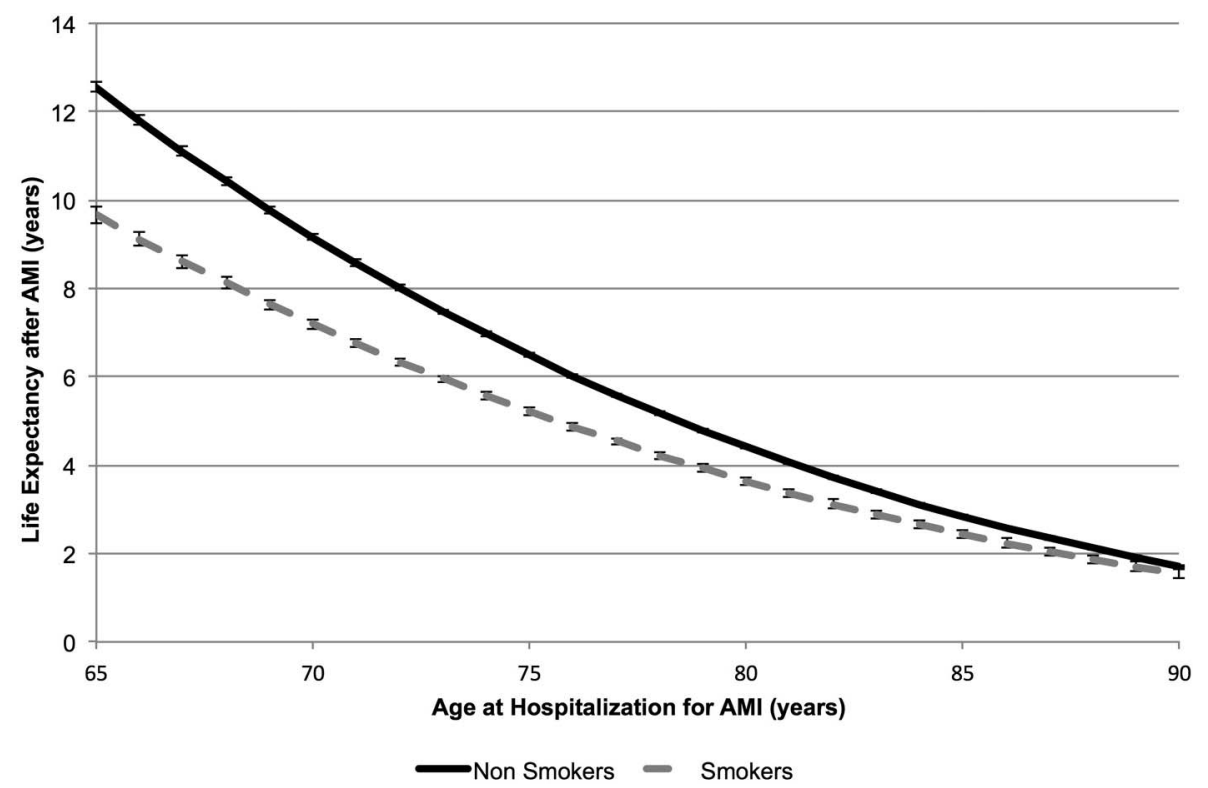

Figure 3 Life expectancy estimates for smokers and non-smokers calculated from admission. Estimates are calculated from a Cox proportional hazards model that includes only age, smoking status and their interaction. AMI, acute myocardial infarction.

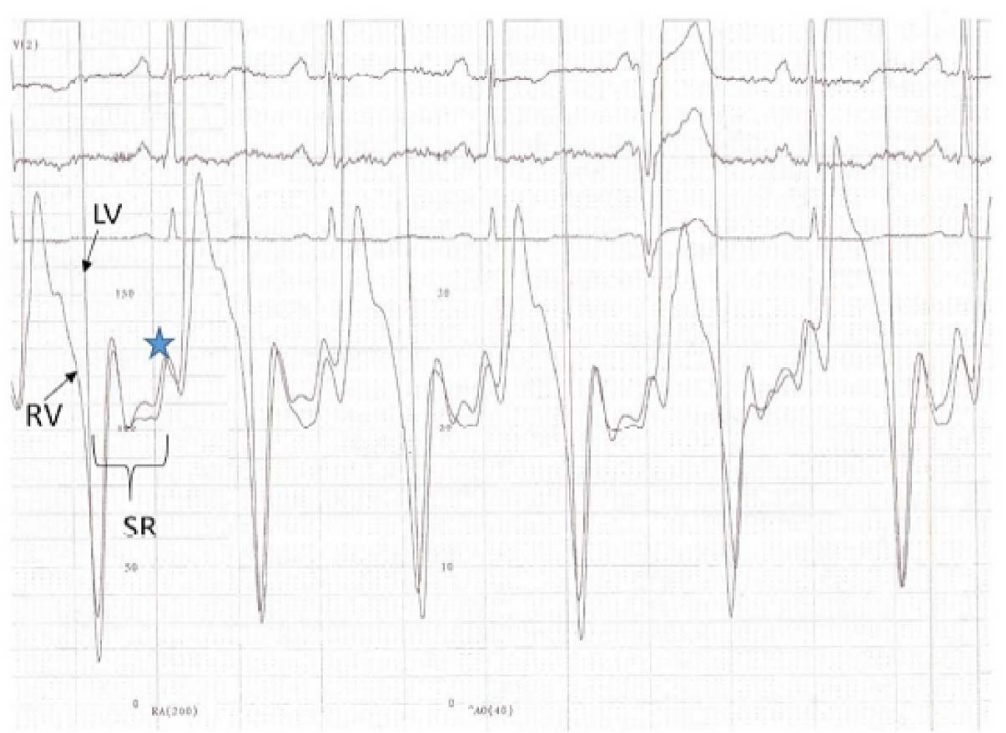

Figure 4 Simultaneous left ventricular (LV) and right ventricular (RV) pressures taken from a patient with constrictive pericarditis. Note the classical dip and plateau, or square root sign in diastole (SR), and equalisation of LV and RV end diastolic pressures to within $5 \mathrm{mmHg}$ (star).

experiencing MACE. In contrast, a higher cut point of $34 \mathrm{ng} / \mathrm{L}$ in men, reclassified $29(1.7 \%)$ from an elevated to a normal troponin, yet $12(41.4 \%)$ still had one-year MACE (figure 2). The authors conclude that in patients presenting to the Emergency Department with a possible ACS, sex-specific cut points improved risk assessment in women, but not in men. The authors also suggest lowering the cut point for both sexes to ensure a high sensitivity for identifying those at risk of MACE. men. Instead, it may be appropriate to consider using a lower cut-point for both men and women to avoid under-diagnosis of acute myocardial infarction although this likely would result in a 25 to $30 \%$ increase in the prevalence of patients with non-ACS related troponin elevations.

The adverse health effects of tobacco smoking are widely known including a higher risk of coronary disease and acute myocardial infarction (AMI) compared to non-smokers. However, the important of stopping smoking after AMI may not be evident to patients or their health care providers, with previous studies suggesting lower crude mortality after AMI in smokers compared to nonsmokers. An important epidemiologic study in this issue of Heart bring clarity to this apparent paradox. (see page 133) In a study of 23,447 Medicare patients (all age 65 years or older) with AMI, a lower 5-year crude mortality after AMI in current smokers (about 15\% of the total study group) was observed but this difference mostly was related to a lower age at AMI. When adjusted for other clinical variables, longterm mortality was higher (17-year HR 1.19 , 95\% CI 1.17 to 1.20 ) and life expectancy was lower after AMI in smokers compared to nonsmokers, regardless of age at AMI (figure 3). The years of life lost in smokers compared to nonsmokers ranged from 1.96 years for AMI at age 65 years to 0.16 years for AMI at age 85 years. These findings emphasize the importance of smoking cessation after AMI even in elderly patients.

The Eduction in Heart article in this issue (see page 147) reviews the role of right heart catheterization in clinical diagnosis, with a discussion of the findings in specific cardiac conditions such as pulmonary hypertension, valvular heart disease, constrictive pericarditis and restrictive cardiomyopathy. Examples of pressure tracings are provided (figure 4) as well as a summary of the indications for endomyocardial biopsy.

The Image Challenge Case (see page 163) shows an ECG tracing with an interesting finding that will test your diagnostic skills.

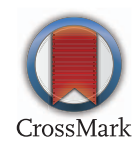

To cite Otto CM. Heart 2016;102:87-88.

Heart 2016;102:87-88.

doi:10.1136/heartjnl-2015-309115 